\title{
A model of very fast oscillations in a realistic cortical axonal plexus
}

\author{
Erin Munro \\ From Nineteenth Annual Computational Neuroscience Meeting: CNS*2010 \\ San Antonio, TX, USA. 24-30 July 2010
}

Very Fast Oscillations (VFOs, $>80 \mathrm{~Hz}$ ) in the hippocampus and cortex have been linked with gap junctions. [1-3]. Modeling work has confirmed that VFOs can be produced by an axon plexus (network of axons connected by gap junctions). [4-6] Here, we explore how a realistic cortical axonal plexus may produce VFOs.

By looking at axonal reconstructions from the rat somatosensory cortex [7], we confirm that an axonal arbor consists of a relatively thick central main axon and collaterals which branch off of the main axon. Considering that myelin is often found on the main axon and not the collaterals [8], we assume that putative gap junctions are placed on axon collaterals. This means that in order for action potentials to travel through an axonal plexus, they must be able to travel up the collaterals, through the main axon, and into the other collaterals. Using simulations, we determine how propagation from one collateral to another is affected by (1) maximum sodium conductance in the axon $\left(\mathrm{g}_{\mathrm{Na}}\right)$, (2) somatic voltage, (3) excitatory chandelier cell synapses onto the initial segment [9], and (4) basket cell synapses onto the soma. We also explore possible network connectivity between collaterals.

The somatic voltage and synapses control propagation between collaterals for a range $\mathrm{g}_{\mathrm{Na}}$. This range depends on (1) how close the collateral is to the soma, and (2) the number of branch points surrounding the junction between the collateral and main axon. We find that many of the collaterals branch off the main axon within 200 um from the soma, so propagation can be controlled by the somatic voltage and synapses in many cells. Furthermore, chandelier and basket cell synapses can interact with each other, so that their overall balance determines propagation between collaterals. Following the work in [4], we piece together the conditions

Correspondence: ecmun@math.bu.com

Mathematics Department, Boston University, Boston, MA, 02215, USA for which propagation occurs in a single axon and the properties of the network to find the conditions under which VFOs occur. We then relate this to the occurrence of VFOs during the up-state of slow-wave sleep, and the lessening of VFOs during the down-state of slow-wave sleep and during waking.

\section{Acknowledgements}

I would like to acknowledge my mentor $\mathrm{N}$ Kopell as well as my former advisor C Börgers for helpful discussions. I would also like to thank neuromorpho.org as well as the labs of H Markram, J Staiger, and K Svoboda for providing axonal reconstructions, and the NSF MSPRF program for support.

Published: 20 July 2010

\section{References}

1. Draguhn A, Traub RD, Schmitz D, Jefferys JG: Electrical coupling underlies high-frequency oscillations in the hippocampus in vitro. Nature 1998, 294:189-192.

2. Grenier F, Timofeev I, Steriade M: Focal synchronization of ripples (80-200 $\mathrm{Hz}$ ) in neocortex and their neuronal correlates. J. Neurophysiol 2001, 86:1884-1898.

3. Schmitz D, Schuchmann S, Fisahn A, Draguhn A, Buhl EH, Petrash-Parwez E, Dermietzel R, Heinemann U, Traub RD: Axon-axonal coupling: a novel mechanism for ultrafast communication. Neuron 2001, 31(5):831-840.

4. Lewis T, Rinzel J: Self-organized synchronous oscillations in a network of excitable cells coupled by gap junctions. Network 2000, 11:299-320.

5. Munro EC: The axonal plexus: a description of the behavior of a network of axons connected by gap junctions. 2008, PhD thesis, Tufts University.

6. Traub RD, Schmitz D, Jefferys JG, Draguhn A: High-frequency population oscillations are predicted to occur in hippocampal pyramidal neuralnetworks interconnected by axo-axonal gap junctions. Neuroscience 1999, 92:407-426.

7. NeuroMorpho.org. [http://neuromorpho.org/neuroMorpho/index.jsp].

8. Sloper JJ, Powell TP: A study of the axon initial segment and proximal axon of neurons in the primate motor and somatic sensory cortex. Philos Trans R Soc Lond B Biol Sci 1979, 285(1006):173-197.

9. Szabadics J, Varga C, Molnár G, Oláh S, Barzó P, Tamás G: Excitatory effect of GABAergic axo-axonic cells in cortical microcircuits. Science 2006, 311(5758):233-235.

doi:10.1186/1471-2202-11-S1-P13

Cite this article as: Munro: A model of very fast oscillations in a realistic cortical axonal plexus. BMC Neuroscience 2010 11(Suppl 1):P13. 